\title{
Current and Emerging Therapies for Gout
}

Samya Mohammad, $D 0^{1}$

Stephanie L. Giattino, $M D^{2}$

Robert T. Keenan, $M D, M P H^{1, *}$

\author{
Address \\ ${ }^{*}$ DDivision of Rheumatology, Duke University School of Medicine, Durham, NC, \\ USA \\ Email: robert.keenan@duke.edu \\ ${ }^{2}$ Department of Internal Medicine, Duke University School of Medicine, 200 Trent \\ Drive DUMC, Durham, NC 3544, USA
}

Published online: 27 February 2015

(C) Springer International Publishing AG 2015

This article is part of the Topical Collection on Gout

Keywords Gout - Hyperuricemia - Urate-lowering therapy - Anti-inflammatory therapy - Allopurinol • Febuxostat . Probenecid - Ulodesine - Levotofisopam - KUX-1151 - RLBN1001 - Arhalofenate - Adrenocorticotropic hormone

\section{Opinion statement}

Gout is the most common inflammatory arthropathy in the western world. It affects millions yearly and accounts for significant disability, lost wages, and increased health care costs. Despite it being a very "curable" disease, it continues to be inadequately treated and many times underestimated as a contributor to the overall disease state. Controlling the acute flares has been historically the priority in the management of gout. Approved therapies to treat acute flares have limitations, especially with gout patients who frequently have multiple comorbidities. Over the last decade, there has been a better understanding that just focusing on the treatment of acute flares, although important, may result in inadequate control of hyperuricemia, resulting in a significant urate burden, chronic arthropathy, and significant disability. Successfully treating gout involves a multi-pronged approach: first, by controlling flares with prophylactic anti-inflammatory medications such as colchicine and non-steroidals and secondly, by treating to target, lowering the serum urate level below $6 \mathrm{mg} / \mathrm{dL}$ (and in some cases $<5,<4$, or even less than $3 \mathrm{mg} / \mathrm{dL}$ is necessary) with uricostatic medications alone or in combination with uricosurics. A greater understanding of the pathophysiology of gout has resulted in the discovery of new therapies to treat and prevent gout flares and underlying hyperuricemia. Novel therapies that lower serum urate levels or treat and prevent acute gouty flares can not only directly improve the care of gout patients but they can also provide the springboard for discourse and the edification of those who treat and those who are treated for this underestimated disease. 


\section{Introduction}

Gout is a crystal-induced inflammatory arthritis that occurs in the setting of elevated serum urate levels, resulting in deposition of monosodium urate (MSU) crystals in articular and periarticular tissues [1]. Hyperuricemia is defined as any level above $6.8 \mathrm{mg} / \mathrm{dL}$, as it exceeds the soluble concentration of urate in body fluid above this level under physiologic conditions. The overall prevalence of gout in the USA is $3.9 \%$, with an estimated 8.3 million adults affected [2]. The prevalence of hyperuricemia alone amongst US adults is $21.4 \%$ or about 43.3 million individuals [2]. Despite the rising prevalence, current therapies are often limited due to side effects, conflicting comorbidities (such as cardiovascular disease, diabetes mellitus, hypertension, chronic kidney disease, metabolic syndrome), and drug-drug interactions [3].

Fortunately, our understanding of the pathophysiology of gout at a molecular level has evolved greatly over the past few decades. Gout is a disease of two intersecting but distinct processes: (1) the intrinsic formation of uric acid, at levels sufficient to drive the precipitation of monosodium urate into crystallized forms and (2) an inflammatory response to the formed crystals [4]. The treatment of acute gout addresses the latter, while the definitive treatment of hyperuricemia and gout targets the former. Traditionally, lowering serum urate levels via inhibiting uric acid synthesis de novo has been the preferred approach with xanthine oxidase inhibitors such as allopurinol and more recently febuxostat. While treating acute gout has historically taken more of a shotgun anti-inflammatory approach, insight into the role of colchicine on the NLRP-3 inflammasome has enabled clinicians to use safer prescribing practices and allowed researchers to consider more target-specific approaches. A better understanding of the NLRP-3 inflammasome, a multi-molecular signaling protein complex in leukocytes, has provided greater insight into the inflammatory response MSU crystals ignite. Inflammation occurs when MSU crystals interact with tissue macrophages in the synovium, triggering activation of the NLRP-3 inflammasome [5]. This complex employs the enzyme caspase- 1 to convert the pro-form of IL- $1 \beta$ into the active form. Secretion of the cytokine IL-1 $\beta$ is thought to be integral in the development of gouty arthritis by causing upregulation of adhesion molecules on vascular endothelial cells and activating signaling pathways that ramp up the inflammatory cascade $[6 \bullet, 7,8]$.

A greater understanding of the pathogenesis of gout over the last decade has provided the impetus for new, more specific therapeutic targets and has finally led to the first approved urate-lowering therapy (ULT) in over four decades with febuxostat. Now, there are several exciting urate-lowering therapies in development, including lesinurad, ulodesine, levotofisopam, KUX-1151, RLBN1001, RDEA3170, and arhalofenate, the latter having both urate lowering and anti-inflammatory properties (Figs. 1 and 2). New therapies for the treatment of inflammation in acute gout have also been studied, including biologics such as anakinra, canakinumab, and rilonacept. Additionally, other anti-inflammatory agents such as corticotropin and melanocortins and caspase inhibitors may be on the horizon for prophylaxis and treatment of acute flares in the future. This review discusses some of the newer medications that may become the future of gout therapy.

\section{Current urate-lowering therapies}

Management of gout involves treatment of the acute inflammation in a joint and the use of serum urate (sUA) lowering therapy to decrease uric acid levels below $6 \mathrm{mg} / \mathrm{dL}$ or lower in some patients [9]. Although high-quality evidence regarding the effect of diet on urate lowering and gout is lacking, diet and lifestyle changes are recommended and remain a cornerstone of therapy [10 $]$. Even when diet and lifestyle changes are implemented, the vast majority of patients still require additional therapy to lower sUA levels to target, so to rid their urate burden and thereby end gout flares [11]. The current FDA approved urate-lowering agents include allopurinol, febuxostat, probenecid, and pegylated uricase (pegloticase). 


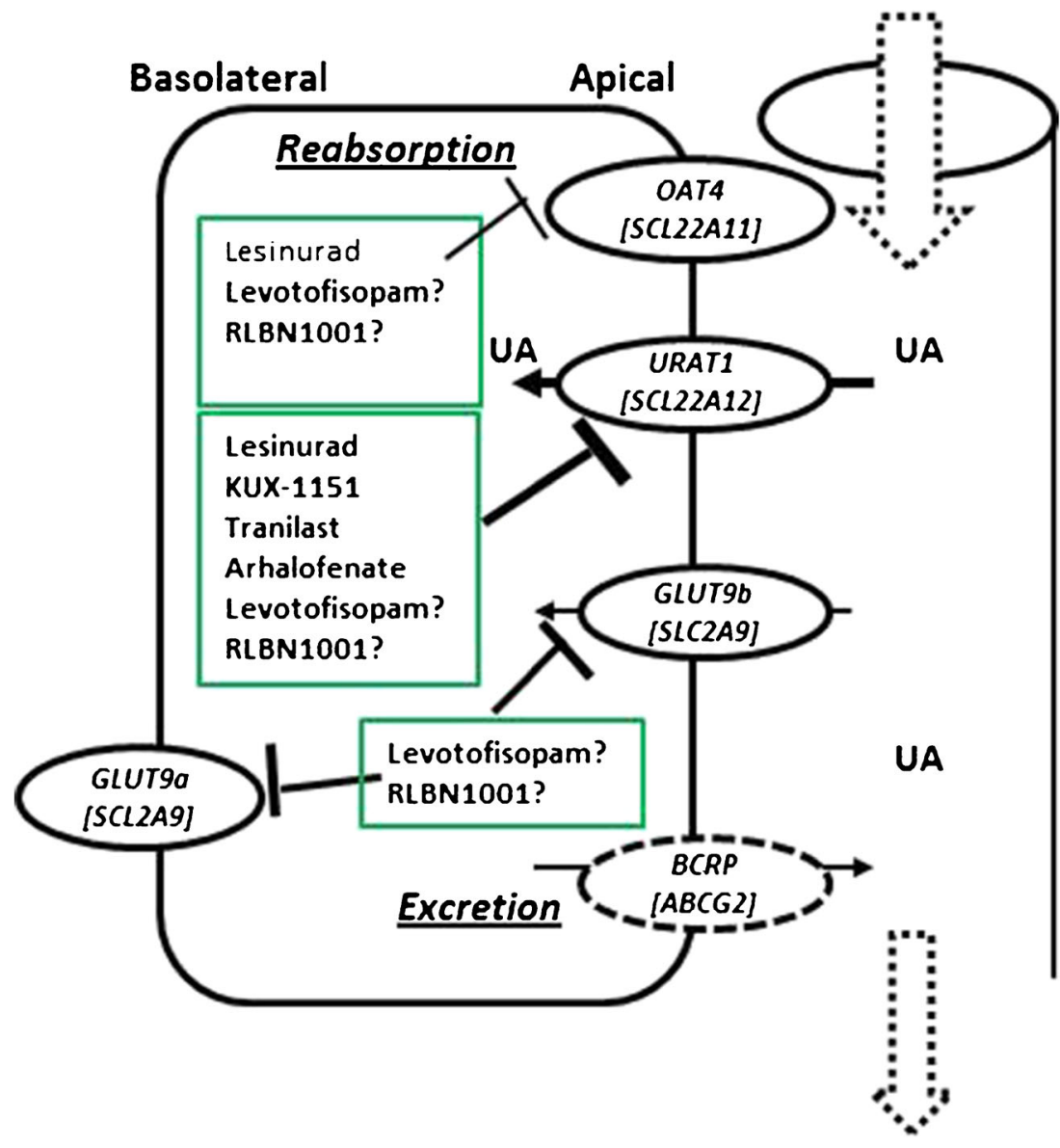

Fig. 1. Summary of uric acid metabolism in the kidney. Specific transporters on the renal tubule epithelial cells contribute to reabsorption of uric acid back into the blood stream or excretion of uric acid in the urine. Novel urate-lowering therapies are designed to target these specific transporters as shown above, increasing urate excretion. UA uric acid, OAT4 organic anion uptake transporter-4, URAT1 urate anion exchanger-1, GLUT9b glucose transporter $9 \mathrm{~b}, B C R P$ breast cancer resistance protein

Allopurinol was approved in 1966 in doses up to $800 \mathrm{mg}$ daily for treatment of gout and still today continues to the best most frequently used ULT [11] and remains a first line therapy in the management of gout [10•]. Allopurinol is a purine analog (isomer of hypoxanthine) that works via inhibition of xanthine oxidase to reduce production of uric acid. Febuxostat is a newer xanthine oxidase inhibitor approved in 2009 by the FDA at 40 and $80 \mathrm{mg}$ daily $[12 \bullet, 13]$. It is a non-purine inhibitor of xanthine oxidase, and unlike allopurinol and its metabolites, febuxostat is not excreted through the kidneys [14].

Although xanthine oxidase inhibitors are the ULT of choice in chronic gout, uricosuric agents, such as probenecid (and others not approved in the United States such as sulfinpyrazone (Anturane) and benzbromarone) were introduced for the treatment of gout before allopurinol [15]. Initially developed for inhibiting the renal tubular secretion of penicillin in the mid-1940s, probenecid was discovered around 1950 to be a uricosuric and beneficial in lowering sUA in patients with gout [16]. Doses range from $250 \mathrm{mg}$ daily to $1000 \mathrm{mg}$ twice daily, and alkalization with sodium bicarbonate or potassium citrate may be required. Probenecid may be useful in reaching sUA targets as combination therapy with allopurinol or febuxostat. The use of probenecid is limited as it 


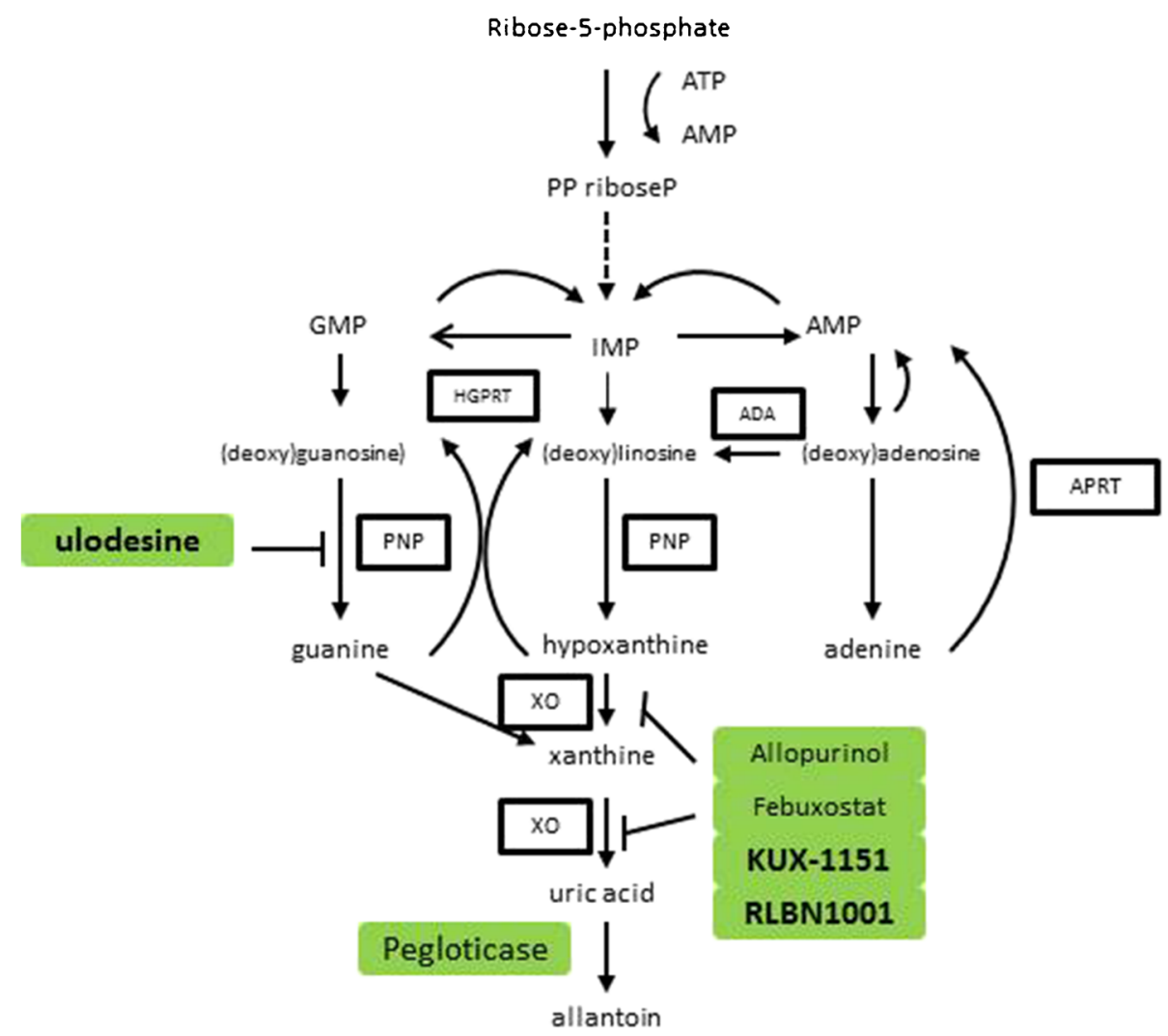

Fig. 2. Uric acid metabolism pathway. Allopurinol, febuxostat, ulodesine, and pegloticase work by decreasing serum urate production as shown. ATP adenosine triphosphate, AMP adenine monophosphate, $P P$ ribose $P$ phospho-ribosyl-pyrophosphate synthetase, IMP inosine monophosphate, GMP guanosine monophosphate, HGRT hypoxanthine-guanine phosphoribosyltransferase, ADA adenosine deaminase, PNP purine nucleoside phosphorylase, xo xanthine oxidase, APRT adenine phosphoribosyltransferase.

\section{Current anti-inflammatory therapies}

increases the risk of urolithiasis, has known drug-drug interactions, and is most efficacious in individuals with GFR $>50 \mathrm{~mL} / \mathrm{min}[12 \bullet]$.

Until recently, FDA-approved ULTs fell into two categories, oral xanthine oxidase inhibitors and oral uricosurics. In September 2010, pegloticase was approved by the FDA for the treatment of hyperuricemia in patients with gout who have failed to normalize sUA levels $(<6 \mathrm{mg} / \mathrm{dL})$ or continue to have signs and symptoms of gout on standard oral ULT [12•]. Pegloticase is an intravenously administered recombinant mammalian urate oxidase (uricase) produced from a genetically modified Escherichia coli conjugated to multiple strands of monomethoxypolyethyl glycol (PEG) [17, 18]. Unlike other available ULT, pegloticase is unique in that it catalyzes the oxidation of uric acid into the more water soluble allantoin, allowing for easy excretion by the kidney [12•].

The long-standing drugs used as prophylaxis when initiating ULT and to treat the inflammation of acute gout attacks include NSAIDs, colchicine, and 
glucocorticoids. These are all considered first line therapies for acute gout, and combination therapy can be used for severe attacks [19•]. Regardless of which medication(s) is chosen, it is recommended that anti-inflammatory therapy be initiated within the first $24 \mathrm{~h}$ of the onset of an attack and most clinicians recommend starting at the very onset of symptoms [19•].

\section{Non-steroidal anti-inflammatory drugs (NSAIDs)}

\section{Colchicine}

NSAIDs have been considered amongst the first line therapy in acute gout treatment and prophylaxis. Drugs that have been approved by the FDA for the use of acute treatment at full dose include naproxen, indomethacin, and sulindac [12•]. Treatment is to be used until the acute attack completely resolves. Other NSAIDs or analgesics may also be effective; there is no consensus recommendation of one NSAID over another $[12 \bullet, 20]$. Unfortunately, NSAIDs can cause both GI and renal toxicity which may preclude its use in a subpopulation of patients. COX-2 selective inhibition may provide an alternative approach; however, its use is also limited due to the potential cardiovascular risks that may be associated.

\section{Glucocorticoids}

Colchicine is an anti-mitotic alkaloid that binds to the cytoskeletal protein tubulin, resulting in the disruption of microtubule polymerization [6•]. Studies have shown pharmacological concentrations of colchicine causing inhibition of intracellular signaling molecules in neutrophils and also inhibiting neutrophil migration; other observations have noted a change in the expression of endothelial adhesion molecules on cells, which are required in the recruitment of neutrophils [6•]. Interestingly, high concentrations of colchicine have been shown to suppress inflammation by blocking IL-1 $\beta$ processing in monocytes stimulated by MSU crystals $[6 \bullet]$. Limitations include drug-drug interactions, particularly those that inhibit the cytochrome P450 pathway and in patients with renal dysfunction.

Glucocorticoids have been used as an alternative therapy for acute gouty arthritis when it is either inappropriate or intolerable to use NSAIDs or colchicine [11]. The anti-inflammatory actions of corticosteroids have been well studied. Intra-articular or oral corticosteroids are recommended for one or two joints involved, and oral corticosteroids can be considered for polyarticular joint involvement [19•]. The dosages range from 30 to $60 \mathrm{mg}$ prednisone daily with a taper over 10-14 days [19•]. In contrast, low-dose glucocorticoids ( $\leq 10 \mathrm{mg} /$ day) can be used as prophylaxis in those patients with an intolerance, contraindication, or refractoriness to NSAIDs and colchicine. The side effects of glucocorticoid use are numerous but most notable including hyperglycemia, hypertension, dyslipidemia, fluid retention, psychosis and agitation $[6 \bullet]$. 
Table 1. Summary of novel urate-lowering therapies in treatment of gout

$\begin{array}{ll}\text { Therapy } & \text { Mechanism of Action } \\ \text { Lesinurad } & \text { Selective uric acid reabsorption inhibitor; inhibits URAT-1 and 0AT4. } \\ \text { Ulodesine } & \text { Decreases serum urate production; purine nucleoside phosphorylase (PNP) inhibitor. } \\ \text { Levotofisopam } & \text { Uricosuric effect. } \\ \text { KUX-1151 } & \text { Dual MOA: decreases serum urate production by xanthine oxidase inhibition and uricosuric } \\ & \text { via URAT-1 inhibition. } \\ \text { RLBN1001 } & \text { Dual MOA: uricosuric via inhibition of URAT-1 and decreases serum urate production. } \\ \text { Arhalofenate } & \text { Dual MOA: uricosuric via inhibition of URAT-1, 0AT4, and 0AT10; also anti-inflammatory } \\ & \text { effect (blocks crystal-induced upregulation of IL-1ß). }\end{array}$

\section{Emerging urate-lowering therapies}

Despite gout being a disease of antiquity, unfortunately it still continues to be an undertreated disease [3]. This is in part not only due to lack of proper use of available medications but also due to the side effects or perceived side effects limiting their use. Many of the anti-inflammatory agents used to treat an acute gout flare cannot be safely used in the presence of comorbid conditions such as renal disease, cardiovascular disease, and diabetes. The future of acute gout treatment, therefore, requires medications that target specific areas of the inflammatory cascade, limiting unwanted and sometimes dangerous adverse effects (Table 1). In addition to the limitations of drug-drug interactions and comorbid conditions, lowering sUA and treating or "curing" gout will not only require additional ULT in the armamentarium but also a greater understanding and additional education of providers and patients to further the understanding of gout, treating to target and the implication of chronic gout on overall health and morbidity.

\section{Lesinurad}

Lesinurad is a selective uric acid reabsorption inhibitor (SURI) that inhibits urate transporter-1 (URAT1) in renal tubules, thereby normalizing uric acid excretion and reducing sUA levels (Fig. 1). This drug also minimally inhibits organic anion transporter 4 (OAT4), a URAT that is enhanced by the use of diuretics $[8,21 \bullet \bullet]$.

A multi-center open label trial investigating the efficacy and safety of lesinurad

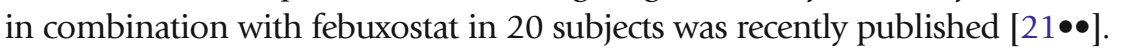
Individuals with baseline sUA $>8 \mathrm{mg} / \mathrm{dL}$ were assigned to two groups, febuxostat 40 or $80 \mathrm{mg}$. Urate levels were checked after receiving febuxostat alone for 1 week. The second week, lesinurad was added at dose $400 \mathrm{mg} /$ day for 1 week and then increased to $600 \mathrm{mg} /$ day the following week. There was a significant decrease in sUA with once daily administration of lesinurad in combination with either febuxostat 40 or $80 \mathrm{mg} /$ day as compared to a single dose febuxostat 40 or $80 \mathrm{mg} /$ day. No serious adverse effects occurred in the study, and all patients

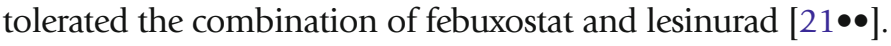


Phase III studies, CLEAR 1 and CLEAR 2 (Combination Study of Lesinurad in Allopurinol Standard of Care Inadequate Responders) were recently completed and published in abstract form at the 2014 American College of Rheumatology Annual Meeting [22]. These were two replicate 12-month, multicenter, randomized, double-blind, placebo-controlled, clinical trials of the efficacy and safety of lesinurad in combination with allopurinol in gout patients with inadequate response to allopurinol.

The studies evaluated lesinurad at 200 or $400 \mathrm{mg}$ oral, once daily, in combination with allopurinol versus allopurinol plus placebo in subjects with gout aged $18-85$ years with sUA $\geq 6.5 \mathrm{mg} / \mathrm{dL}$ at screening [22]. Subjects were also required to be on stable doses of allopurinol $\geq 300 \mathrm{mg}$ ( $\geq 200 \mathrm{mg}$ for moderate renal impairment) and have history of $\geq 2$ gout flares in the prior 12 months. The primary endpoint was the proportion of subjects meeting sUA target of $<6.0 \mathrm{mg} / \mathrm{dL}$ by month 6 . Secondary endpoints included mean gout flare rate requiring treatment from months $6-12$ and proportion of subjects with complete resolution of $\geq 1$ target tophus by month 12 .

Lesinurad (200 or $400 \mathrm{mg}$ ) in combination with allopurinol significantly increased the proportion achieving sUA target at 6 months by 2-2.5-fold compared to allopurinol alone [22]. Regarding adverse events, with the exception of a higher incidence in predominately reversible serum creatinine elevations, lesinurad at the $200 \mathrm{mg}$ dose was comparable to allopurinol alone. The $400 \mathrm{mg}$ dose also had a higher incidence of increase in serum creatinine levels, a small percentage of which did not reverse while still on therapy. Interestingly, lesinurad did not appear to increase the risk of renal stones. Combination therapy with lesinurad and a xanthine oxidase inhibitor may represent a future treatment option for gout patients who have not achieved their sUA target.

In addition to lesinurad, Ardea Biosciences may have another potent uricosuric in clinical trials soon. RDEA3170 was found to have a high affinity for URAT1 and greater urate-lowering effect compared to other known uricosurics [23].

\section{Ulodesine}

Ulodesine, developed by BioCryst Pharmaceuticals, is a purine nucleoside phosphorylase (PNP) inhibitor (Fig. 2) with once daily oral dosing that is currently under clinical trials [11]. It acts upstream of xanthine oxidase in the purine metabolism pathway to reduce serum urate production $[8,24]$. In phase 2 placebo-controlled trials, ulodesine in doses ranging from 40 to $80 \mathrm{mg}$ once daily resulted in lower sUA levels in conjunction with allopurinol in patients who have not responded alone to allopurinol $300 \mathrm{mg}$ daily [25]. Another phase 2 trial tested the addition of low-dose ulodesine to various doses of allopurinol. It was observed that $100 \%$ of subjects taking ulodesine 40 and $80 \mathrm{mg} /$ day in combination with $300 \mathrm{mg} /$ day of allopurinol achieved the target of serum uric acid less than $6.0 \mathrm{mg} / \mathrm{dL}$ [11]. The higher doses were noted to have more diarrhea and rash compared to the placebo group. Notably, no increase in infection was noted, which would be a concern, since inborn error of purine metabolism from PNP deficiency is associated with a combined 


\section{Levotofisopam}

immunodeficiency [11]. Currently, further studies have not gone forth, so the future of ulodesine is speculative.

KUX-1151

Levotofisopam is the s-enantiomer of the racemic mixture tofisopam, an agent that has been used for the treatment of a variety of disorders associated with stress or autonomic instability and was found to have uricosuric properties. Two phase 1 studies of levotofisopam were conducted by Velos Pharmaceuticals prior to its merger with Pharmos Corporation. The study was done on individuals from UK and Netherlands, and a large, rapid reduction in serum uric acid was noted [11].

An open label phase 2a trial of levotofisopam at the Duke Clinical Research Unit of Duke University enrolled 13 subjects, with screening serum uric acid levels between 8 and $12 \mathrm{mg} / \mathrm{dL}$ [26]. Each subject received a single dose of $50 \mathrm{mg}$ on days 1 and 7 and and $50 \mathrm{mg}$ two times a day for days 2-6. All 13 subjects achieved the target urate level less $6.0 \mathrm{mg} / \mathrm{dL}$, while 77 and $54 \%$ achieved the targets of less than 5.0 and less than $4.0 \mathrm{mg} / \mathrm{dL}$, respectively [11, 26]. Treatment of gout patients with levotofisopam for 7 days was well tolerated with no significant laboratory abnormalities and no unexpected, serious, or severe adverse events [26].

RLBN1001

Pfizer's KUX-1151 is a new agent that has completed a phase I clinical trial in Japan and began recruiting for a phase II trial in the USA in 2014. KUX-1151 is another promising and novel investigational ULT. It has a dual mechanism of action and reduces serum uric acid levels through inhibiting both xanthine oxidase and uric acid transporter (URAT1) [11].

RLBN1001 was initially developed as a prototype anti-cancer drug and found to cause marked hypopuricemia in a study of 350 human subjects [27]. A proofof-concept study in 50 human subjects treated with RLBN1001 found that at both low and high doses, hypouricemia was associated with increased urinary excretion of both uric acid and total oxypurines, suggesting bifunctional equilibrium effects on production and excretion of urate. The investigators found that RLBN1001's effect on decreasing production was 4-fold more potent than allopurinol and its inhibition of URAT1 was equipotent to lesinurad [27].

\section{Arhalofenate}

Arhalofenate, a peroxisome proliferator-activated receptor gamma (PPAR- $\gamma$ ) partial agonist initially developed to treat diabetes, was discovered to have 
properties advantageous to the treatment of acute and chronic gout while this drug was being developed for glycemic control by Cymabay Therapeutics [11, $28,29]$. The decreases in serum uric acid are due to a uricosuric effect, in which transporters URAT-1, OAT4, and OAT10 are blocked by arhalofenate, preventing the reabsorption of uric acid in the renal tubes and allowing for increased renal clearance [30 $[$. Interestingly, arhalofenate was also observed to inhibit MSU crystal induced upregulation of IL-1 $\beta$ which provides a benefit in acute management of gouty inflammation as it may provide simultaneous ULT and prophylactic effects. This drug has now undergone three phase $2 \mathrm{a}$ studies and currently clinical phase $2 \mathrm{~b}$ trial is underway [30 $]$.

\section{Emerging therapies for the prophylaxis and treatment of acute gout anakinra}

As a soluble recombinant IL-1 $\beta$ receptor antagonist, anakinra binds to IL-1R1 and blocks IL-1 $\beta$ from binding the receptor and initiating signal transduction. It has a short half-life of 4-6 h and was initially developed for the treatment of rheumatoid arthritis but has now been tested for efficacy of treatment of acute gout, given the role of IL-1 $\beta$ in gouty inflammation $[7,8,11]$.

In an open-label proof-of-concept study, 10 patients with contraindications to or failures of standard anti-inflammatory therapies who were having acute gout flare or recurrent flares on ULT, received anakinra for 3 days. Pain reduction was noted to be rapid and by day 3 a mean reduction in pain of $79 \%$, with complete resolution of $90 \%$ of affected joints on physical exam [8]. In a larger case series, 26 charts were reviewed of hospitalized patients who received anakinra. Improvement in pain was noted within $24 \mathrm{~h}$ of treatment in $67 \%$ of patients, with $72.5 \%$ of patient noting complete resolution by day 5 [31]. There were few adverse events, with the exception of one patient who was found to be unresponsive after repeated treatment with anakinra. Anakinra may find itself useful in the treatment of complex patients who have been refractory or have contraindications to other therapies for acute gout.

\section{Canakinumab and rilonacept}

Canakinumab is a fully humanized anti-IL-1 $\beta$ monoclonal antibody that binds to soluble IL-1 $\beta$ and prevents receptor activation $[8,11]$. One double-blind study assessed the efficacy and safety of one dose of $150 \mathrm{mg}$ canakinumab against one single dose of triamcinolone injection at baseline and during an acute gout attack in patients frequently flaring with contraindications to use of NSAIDs and/or colchicine [32]. This study showed canakinumab to have a rapid onset in pain relief and increased the time between a flare, likely attributable to its half-life of 21-28 days [29, 32]. More adverse events were noted in the canakinumab group, which included infections, neutropenia, and thrombocytopenia [32]. Another study compared canakinumab (single dose versus four divided doses weekly) versus colchicine $0.5 \mathrm{mg}$ daily for gout prophylaxis 
during initiation of ULT with allopurinol [43]. Cankinumab was found to be superior prophylaxis than its comparator colchicine. The use of canakinumab is approved in Europe for patients with contraindications to use of traditional anti-inflammatory medications [11].

Rilonacept is a dimeric fusion protein (or IL-1 Trap) consisting of the extracellular domains of human IL-1 $\beta$ receptor and IL-1 receptor accessory protein fused to the Fc portion of human IgG [8]. In a phase 2 trial, rilonacept (320 mg loading dose and $160 \mathrm{mg}$ weekly) was compared to placebo for prophylaxis while initiating ULT with allopurinol and was found to be superior in reducing gout flares during this period [33]. Other phase 3 trials which compared rilonacept versus placebo found similar results $[33,34]$. Conversely, in a randomized, controlled clinical trial using indomethacin as comparator to rilonacept, rilonacept alone or combined with indomethacin was not significantly superior to indomethacin alone [35]. Rilonacept, with a half-life of 9 days, was overall well tolerated in both studies with adverse reactions noted to be predominately injection site reactions $[35,36]$.

\section{Corticotropin and melanocortins}

Adrenocorticotropic hormone (ACTH) or corticotropin was the first steroid pathway agent used in rheumatic disease and its effectiveness as an anti-inflammatory was presumed to be due to its ability to stimulate adrenal production of corticosterone [7]. ACTH is a precursor molecule for melanocortin, and more recently, it has been appreciated that ACTH binds to all five known melanocortin receptors besides melanocortin-2 receptor, the receptor responsible for adrenal stimulation. Significant in the management of gout, ACTH has activity at melanocortin-3 receptor (MC3R), which belongs to the family of 7-transmembrane G-protein coupled receptors $[7,11]$. When MC3R is bound by either melanocortin or corticotropin, it initiates anti-inflammatory signals sequestering the activities of NF- $\kappa \mathrm{B}$ and heme oxygenase, resulting in decreased inflammatory cytokine transcription and production [11]. This pathway was further explored by Getting and coworkers using a rat model for acute gout $[37,38]$. After corticotropin was injected subcutaneously, increased levels of corticosterone and signs of reduced inflammation were observed. It was also noted that when corticotropin was injected directly into the joint, increased levels of corticosterone were not seen in the setting of decreased inflammation, indicating that ACTH might have antiinflammatory properties independent of glucocorticoid release [37, 38]. A case series reviewed 181 inpatient cases of gout in which ACTH was used as first line treatment [39]. Investigators found that $77 \%$ of patients responded after one intramuscular injection. Regarding the non-responders, $87 \%$ received a second injection the following day, of which $82 \%$ responded [39]. Further clinical trials will be needed to evaluate the efficacy of this treatment, as it would fill a critical need for therapies in 
patients who have contraindications to NSAIDs, colchicine, and possibly high-dose oral corticosteroids.

\section{Caspase inhibitors}

\section{Summary}

Caspases-1-4, through their involvement with inflammasomes, mediate the maturation and secretion of IL-1 [40]. Caspase inhibitors have been shown in murine models to inhibit IL-1; however, only case reports are currently available regarding its efficacy in humans [11]. Whether or not caspase inhibitors come to fruition in gout remains to be seen.

A greater understanding of the pathogenesis of gout over the last decade has provided the impetus for new treatments of this ancient yet debilitating disease. Some promising ULTs for gout such as tranilast (a uricosuric with anti-inflammatory and analgesic properties), ulodesine, and uricase peg-20 (pegylated recombinant uricase derived from Candida utilis used in China) have fallen to the waste side or have been stalled in development. But still, many other ULT such as the uricosurics, lesinurad, and levotofisopam or the dual mechanism of action drugs RLBN1001 and arhalonfenate provide optimism for a larger and more diverse arsenal in gout management. New anti-inflammatory therapies such as the IL-1 inhibitors may better prevent or treat acute flares while lowering sUA levels to target. The implications of using more potent or tolerated antiinflammatory therapies could not only mean decreased or shortened disability during a flare but also better compliance with more potent combination or dual action ULT such as lesinurad or KUX-1151, respectively. Although there are few studies evaluating the cost effectiveness of new versus old gout therapies, there is some evidence that dose escalation and sequential therapy (from old to new) may be cost effective when taking

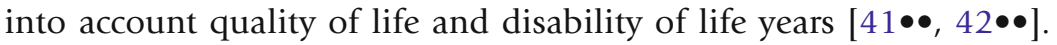

While to date, there have just been two new medications that have materialized and become part of the clinicians arsenal against this malady, they have revitalized the discussion, education, and research into the most common of inflammatory arthropathies. The pipeline of gout therapeutics can rejuvenate the care of gout patients by illuminating the comorbidities that are associated with it and, further, raise the bar in how it is managed. New guidelines have been introduced regarding the management of gout across the globe, while a new staging system has been proposed that goes beyond the traditional symptomology stages designated as hyperuricemia, acute intermittent gout, and advanced gout [30•]. Additionally, measuring and treating to target is expected to become part of meaningful use in everyday practice. Future research and future therapies can provide the basis for dialogue and pedagogical strategies to change the arguably harmful dogma that is at the root of 'when it hurts treat it and if it doesn't don't' and further illustrate the fact that gout can be cured. 


\section{Compliance with Ethics Guidelines}

\section{Conflict of Interest}

Samya Mohammad and Stephanie L. Giattino declare that they have no conflict of interest.

Robert T. Keenan has received advisory board payments from Takeda, Crealta, and AstraZeneca.

\section{Human and Animal Rights and Informed Consent}

This article does not contain any studies with human or animal subjects performed by any of the authors.

\section{References and Recommended Reading}

Papers of particular interest, published recently, have been highlighted as:

- Of importance

- Of major importance

1. Perez-Ruiz F, Liote F. Lowering serum uric acid levels: what is the optimal target for improving clinical outcomes in gout? Arthritis Rheum. 2007;57(7):1324-8.

2. Zhu Y, Pandya BJ, Choi HK. Prevalence of gout and hyperuricemia in the US general population: the National Health and Nutrition Examination Survey 2007-2008. Arthritis Rheum. 2011;63(10):3136-41.

3. Keenan RT et al. Prevalence of contraindications and prescription of pharmacologic therapies for gout. Am J Med. 2011;124(2):155-63.

4. Firestein GS, Kelley WN. Kelley's textbook of rheumatology. Philadelphia: Elsevier/Saunders; 2013.

5. Martinon F et al. Gout-associated uric acid crystals activate the NALP3 inflammasome. Nature. 2006;440(7081):237-41.

6. Cronstein BN, Sunkureddi P. Mechanistic aspects of inflammation and clinical management of inflammation in acute gouty arthritis. J Clin Rheumatol. 2013;19(1):19-29.

A good review of the pathophysiology of acute gout.

7. Pillinger MH, Keenan RT. Update on the management of hyperuricemia and gout. Bull NYU Hosp Jt Dis. 2008;66(3):231-9.

8. Crittenden DB, Pillinger MH. New therapies for gout. Annu Rev Med. 2013;64:325-37.

9. Seth R et al. Allopurinol for chronic gout. Cochrane Database Syst Rev. 2014;10:Cd006077.

10. Khanna D et al. American College of Rheumatology guidelines for management of gout. Part 1: systematic nonpharmacologic and pharmacologic therapeutic approaches to hyperuricemia. Arthritis Care Res (Hoboken). 2012;64(10):1431-46.

Part 1 of the treatment guidelines for gout published by the ACR.

11. Edwards NL, So A. Emerging therapies for gout. Rheum Dis Clin North Am. 2014;40(2):375-87.
12. Keenan RT. Safety of urate-lowering therapies: managing the risks to gain the benefits. Rheum Dis Clin North Am. 2012;38(4):663-80.

A thorough review of the currently approved urate lowering therapies as well as potential side effects and contraindications one may encounter with gout patients.

13. Becker MA et al. The urate-lowering efficacy and safety of febuxostat in the treatment of the hyperuricemia of gout: the CONFIRMS trial. Arthritis Res Ther. 2010;12(2):R63.

14. Keenan RT, Pillinger MH. Febuxostat: a new agent for lowering serum urate. Drugs Today (Barc). 2009;45(4):247-60.

15. Zhang W et al. EULAR evidence based recommendations for gout. Part II: Management. Report of a task force of the EULAR Standing Committee for International Clinical Studies Including Therapeutics (ESCISIT). Ann Rheum Dis. 2006;65(10):1312-24.

16. Boger WP, Strickland SC. Probenecid (benemid); its uses and side-effects in 2,502 patients. AMA Arch Intern Med. 1955;95(1):83-92.

17. Sundy JS et al. Pharmacokinetics and pharmacodynamics of intravenous PEGylated recombinant mammalian urate oxidase in patients with refractory gout. Arthritis Rheum. 2007;56(3):10218 .

18. Sundy JS et al. Efficacy and tolerability of pegloticase for the treatment of chronic gout in patients refractory to conventional treatment: two randomized controlled trials. JAMA. 2011;306(7):711-20.

19. Khanna D et al. American College of Rheumatology guidelines for management of gout. Part 2: Therapy and antiinflammatory prophylaxis of acute gouty arthritis. Arthritis Care Res (Hoboken). 2012;64(10):1447-61. 
Part 2 of the American College of Rheumatology treatment guidelines.

20. van Durme CM et al. Non-steroidal anti-inflammatory drugs for acute gout. Cochrane Database Syst Rev. 2014;9:Cd010120.

21.• Fleischmann R et al. Pharmacodynamic, pharmacokinetic and tolerability evaluation of concomitant administration of lesinurad and febuxostat in gout patients with hyperuricaemia. Rheumatology (Oxford). 2014;53(12):2167-74.

A recently published trial of the pipeline selective uricosuric lesinurad.

22. Saag K, Adler S, Bhakta N, Fung M, Kopicko J, Storgard C, Bardin T. Lesinurad, a Novel Selective Uric Acid Reabsorption Inhibitor, in Two Phase III Clinical Trials: Combination Study of Lesinurad in Allopurinol Standard of Care Inadequate Responders (CLEAR 1 and 2) in American College of Rheumatology Annual Meeting. Boston, MA; November 14-19 2014.

23. Miner J, Tan P. RDEA3170 a novel high affinity URAT1 inhibitor binds to central domain within URAT1. Ann Rheum Dis. 2012;71 Suppl 3:446.

24. Fitz-Patrick DDW, Pappas J, Hollister A. Effects of a purine nucleoside phosphorylase inhibitor, BCX4208, on the serum uric acid concentrations in patients with gout. [abstract]. Arthritis Rheum. 2010;62 Suppl 10:150.

25. Hollister AS, Becker MA, Terkeltaub R, Waugh A, Lyman S, Flynt A, et al. BCX4208 synergistically lowers serum uric acid (sUA) levels when combined with allopurinol in patients with gout: results of a phase 2 dose-ranging trial. [abstract]. Arthritis Rheum. 2011;63 Suppl 10:1018.

26. Pharmos Corporation Announces Successful Completion of Proof-of-concept clinical trial of levotofisopam for the treatment of gout May 17, 2012, PR Newsire: Iselin, NJ.

27. Warrell RP, Klukovits A, Barnes K, et al. Profound hypouricemia induced in human subjects by novel bifunctional inhibitors of xanthine oxidase and URAT1. Arthritis Rheum. 2014;66(11 (Suppl)):S366.

28. CymaBay Initiates Arhalofenate Phase $2 \mathrm{~b}$ Gout Study. March 26, 2014: Newark, CA. http://www. marketwired.com/press-release/cymabay-initiatesarhalofenate-phase-2b-gout-study-otcqb-cyma1892852.htm. Accessed 3 Jan 2015.

29. Saha GC, Karpf D, Choi YJ, Roberts BK. Arhalofenate, a potential novel treatment for hyperuricemia, with or without metabolic co-morbidities, in patients with gout: meta-analysis of urate lowering in four phase 2 studies in type 2 diabetes [abstract]. Arthritis Rheum. 2011;63 suppl 10:2584.

30. Dalbeth N, Stamp L. Hyperuricaemia and gout: time for a new staging system? Ann Rheum Dis. 2014;73(9):1598-600.

A newly proposed criteria for gout staging based upon the pathogenesis of gout. The new criteria would provide a focus on gout as a chronic disease of MSU crystal deposition, and emphasize the importance of targeting the underlying basis of disease in order to achieve dissolution of MSU crystals and 'cure' of gout.

31. Ghosh P et al. Treatment of acute gouty arthritis in complex hospitalized patients with anakinra. Arthritis Care Res (Hoboken). 2013;65(8):1381-4.

32. Schlesinger $\mathrm{N}$ et al. Canakinumab for acute gouty arthritis in patients with limited treatment options: results from two randomised, multicentre, active-controlled, double-blind trials and their initial extensions. Ann Rheum Dis. 2012;71(11):1839-48.

33. Schumacher Jr HR et al. Rilonacept (interleukin-1 trap) in the prevention of acute gout flares during initiation of urate-lowering therapy: results of a phase II randomized, double-blind, placebo-controlled trial. Arthritis Rheum. 2012;64(3):876-84.

34. Mitha $\mathrm{E}$ et al. Rilonacept for gout flare prevention during initiation of uric acid-lowering therapy: results from the PRESURGE-2 international, phase 3, randomized, placebo-controlled trial. Rheumatology (Oxford). 2013;52(7):1285-92.

35. Terkeltaub RA et al. Rilonacept in the treatment of acute gouty arthritis: a randomized, controlled clinical trial using indomethacin as the active comparator. Arthritis Res Ther. 2013;15(1):R25.

36. Schumacher Jr HR et al. Rilonacept (interleukin-1 trap) for prevention of gout flares during initiation of uric acid-lowering therapy: results from a phase III randomized, double-blind, placebo-controlled, confirmatory efficacy study. Arthritis Care Res (Hoboken). 2012;64(10):1462-70.

37. Getting SJ et al. Activation of melanocortin type 3 receptor as a molecular mechanism for adrenocorticotropic hormone efficacy in gouty arthritis. Arthritis Rheum. 2002;46(10):2765-75.

38. Getting SJ et al. Melanocortin 3 receptors control crystal-induced inflammation. FASEB J. 2006;20(13):2234-41.

39. Daoussis D et al. ACTH as first line treatment for acute gout in 181 hospitalized patients. Joint Bone Spine. 2013;80(3):291-4.

40. Gross $\mathrm{O}$ et al. Inflammasome activators induce interleukin-1alpha secretion via distinct pathways with differential requirement for the protease function of caspase-1. Immunity. 2012;36(3):388-400.

41.• Beard SM et al. Cost-effectiveness of febuxostat in chronic gout. Eur J Health Econ. 2014;15(5):453-63.

An interesting and helpful report of the pharmacoeconomics in gout treatment.

42.•• Jutkowitz E et al. Cost-effectiveness of allopurinol and febuxostat for the management of gout. Ann Intern Med. 2014;161(9):617-26.

An interesting and helpful report of the pharmacoeconomics in gout treatment.

43. Schlesinger $\mathrm{N}$ et al. Canakinumab reduces the risk of acute gouty arthritis flares during initiation of allopurinol treatment: results of a double-blind, randomised study. Ann Rheum Dis. 2011;70(7):1264-71. 